\title{
Postural Hand Synergies for Tool Use
}

\author{
Marco Santello, Martha Flanders, and John F. Soechting \\ Neuroscience Department, University of Minnesota, Minneapolis, Minnesota 55455
}

Subjects were asked to shape the right hand as if to grasp and use a large number of familiar objects. The chosen objects typically are held with a variety of grips, including "precision" and "power" grips. Static hand posture was measured by recording the angular position of 15 joint angles of the fingers and of the thumb. Although subjects adopted distinct hand shapes for the various objects, the joint angles of the digits did not vary independently. Principal components analysis showed that the first two components could account for $>80 \%$ of the variance, implying a substantial reduction from the 15 degrees of freedom that were recorded. However, even though they were small, higher-order (more than three) principal compo- nents did not represent random variability but instead provided additional information about the object. These results suggest that the control of hand posture involves a few postural synergies, regulating the general shape of the hand, coupled with a finer control mechanism providing for small, subtle adjustments. Because the postural synergies did not coincide with grip taxonomies, the results suggest that hand posture may be regulated independently from the control of the contact forces that are used to grasp an object.

Key words: hand shape; grasping; kinematics; fingers; grip; human
The homunculus has huge hands, meaning that a disproportionate amount of sensorimotor cortex is devoted to hand use. From this observation, one might surmise that there would be a considerable amount of flexibility in generating a variety of hand postures and in the control of the individual joints of the hand. However, to date, most studies have emphasized the contrary, namely the lack of individuation in finger movements (cf. Schieber, 1995).

An early attempt to characterize the posture of the hand for grasping was made by Napier (1956). He defined two distinct patterns of movement, which he called "precision grip" and "power grip." In the former, one or more of the fingers, possibly in opposition to the thumb, make contact with the object and exert pressure on it (Johansson and Cole, 1992). In contrast, in the power grip the palm of the hand also is in contact with the object. Following Napier, numerous investigators (Kamakura et al., 1980; Elliott and Connolly, 1984; Klatzky et al., 1987; Cutkosky and Howe, 1990) have elaborated on this scheme by proposing further subdivisions, such as "prismatic" and "circular" grips, or "tripod," "lateral," and "tip prehensile" grips. In each instance, these subdivisions are based on which constellation of fingers exerts force on the object and which part of the finger (finger pad or lateral aspect) contacts the object. The concept of "virtual fingers" introduced by Iberall and colleagues (Iberall et al., 1986; Iberall and MacKenzie, 1990) is based on similar considerations. In their proposal, each virtual finger comprises all of the fingers that are controlled as a unit to exert force to grasp the object.

All of these studies were based on a consideration of which of the fingers (and thumb) were used to generate force and assumed implicitly that the posture of the hand would be correlated with

\footnotetext{
Received June 29, 1998; revised Aug. 17, 1998; accepted Sept. 15, 1998.

This work was supported by United States Public Health Service Grants NS-15018 and NS-27484.

Correspondence should be addressed to John F. Soechting, Department of Physiology, 6-255 Millard Hall, University of Minnesota, Minneapolis, MN 55455.

Copyright (C) 1998 Society for Neuroscience 0270-6474/98/1810105-11\$05.00/0
}

this criterion. If this is true, then hand posture in grasp should not vary along a continuum, but, rather, there should be a discrete set of postures, each corresponding to one of the grips. We tested this hypothesis by asking subjects to shape the right hand to grasp and use a set of familiar objects and then measuring the resulting configuration of the hand. Hand postures were distributed in a multidimensional continuum, with little evidence for clustering. The dimensionality of the space required to characterize hand posture was considerably smaller than the number of degrees of freedom that were measured (15 degrees of freedom). But, as one might expect from the amount of cortical area devoted to the hand, it was large enough ( $\sim 5$ degrees of freedom) to support the potential of individuated finger movement.

\section{MATERIALS AND METHODS}

Experimental task. Subjects were instructed to shape the right hand to grasp and use a large number of imagined objects $(n=57$; Table 1$)$. They were encouraged to move the proximal arm in tandem with the hand motion. An object was named at the beginning of each trial. We selected objects spanning a large range of sizes and shapes to assess the consequent modulation of hand posture. To allow comparison between our results and previous work, we chose mostly objects that had also been used by other authors to formulate taxonomies of hand postures (Kamakura et al., 1980; Klatzky et al., 1987; Cutkosky and Howe, 1990).

The subject was asked to imagine the object floating in space at a distance of $\sim 40 \mathrm{~cm}$ anterior to the subject's frontal plane. The elbow and wrist rested on a flat surface, the forearm was horizontal, the arm was oriented in the parasagittal plane passing through the shoulder, and the hand was in a semipronated position. At presentation of a "go" signal, the subject moved the arm and hand as if to grasp and use the named object. A contact switch was released at movement onset. When subjects had attained a static hand posture, they pressed a second switch with their left hand. Each subject performed a total of five trials for each of the objects; all trials were presented in random order.

Five right-handed subjects (three males and two females, age ranging from 30 to 41 years) took part in the experiments. All subjects gave informed consent, and the protocols were approved by the Institutional Review Board of the University of Minnesota.

Experimental procedures and analysis. Hand posture was measured by 15 sensors embedded in a glove (CyberGlove; Virtual Technologies, Palo Alto, CA) as described previously (Santello and Soechting, 1997, 1998; 


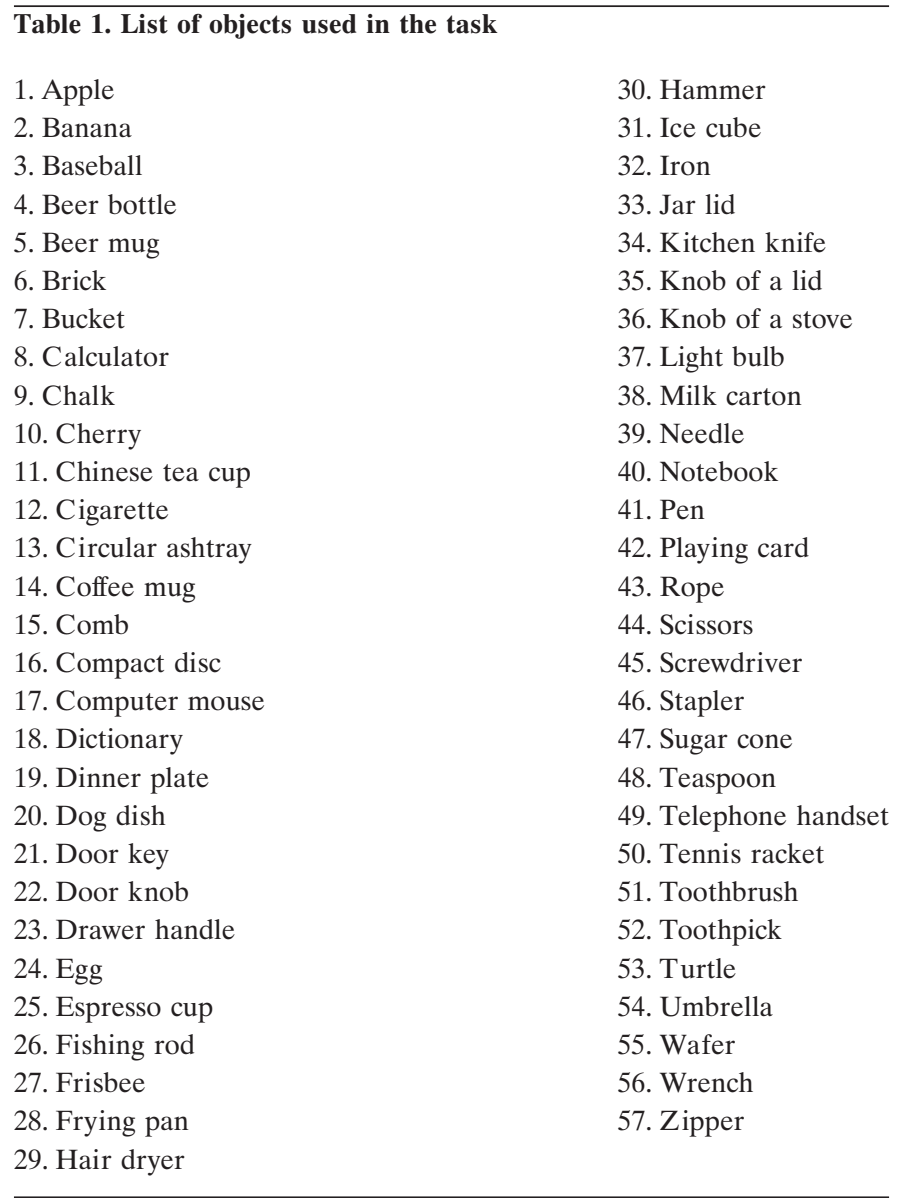

Soechting and Flanders, 1997). We measured the angles at the metacarpalphalangeal (mcp) and proximal interphalangeal (pip) joints of the four fingers and the angle of abduction (abd) between adjacent fingers. For the thumb, the mcp, abd, and interphalangeal (ip) angles were measured, as was the angle of thumb rotation (rot) about an axis passing through the trapeziometacarpal joint of the thumb and index mcp joint. Flexion and abduction were defined as positive; the mcp and pip angles were defined as $0^{\circ}$ when the finger was straight and in the plane of the palm. At the thumb, positive values of thumb rotation denoted internal rotation. The spatial resolution of the CyberGlove was $<0.1^{\circ}$.

The output of the transducers was sampled at $12 \mathrm{msec}$ intervals. The two switches described above were used to determine the onset and termination of the movement. The static hand postures at the end of the movement were analyzed using (1) discriminant analysis, (2) regression analysis, and (3) principal components analysis.

We used discriminant analysis (Johnson and Wichern, 1992) as a means to determine the extent to which hand postures were reliably different for the 57 objects that were named. The procedures used to compute the discriminant functions have been described in detail elsewhere (Santello and Soechting, 1998). In brief, discriminant functions are the linear combinations of the joint angles that maximize the ratio of the between-groups variance to the within-groups variance. In our experiment, each group corresponded to the data sets from the five trials for 1 of the 57 objects. After group means were computed, a given trial was then allocated to the object it was closest to in discriminant space, i.e., the space formed by the discriminant functions.

The results of the discriminant analysis were used to construct a confusion matrix (Sakitt, 1980; Johnson and Phillips, 1981) that provided a summary of the extent to which hand posture on each trial could correctly predict the object that was grasped. Each entry in this matrix corresponded to the number of trials for which an instructed posture (rows) corresponded best to a particular object (columns). If subjects performed perfectly, all entries would be on the diagonal. We then used information theory (Shannon, 1948) to characterize each subject's performance. Specifically, we computed the sensorimotor efficiency (SME) index, defined as the ratio between the information transmitted by hand posture and the maximum possible amount of information that could be transmitted (Sakitt, 1980; Santello and Soechting, 1998). This analysis defined the extent to which hand postures differed for different objects, but it did not provide insight into how the hand was shaped for different objects.

Regression analysis was used as a first step to assess the extent to which the angular excursions of the 15 sensors covaried with each other. Patterns of covariation were further investigated using principal components analysis (Glaser and Ruchkin, 1976). For each subject, the five trials per object were first averaged to obtain a total of 57 hand postures. For each sensor, we then subtracted the grand mean computed over the 57 objects (so that the range was centered about $0^{\circ}$ ). The hand posture for each object was thus characterized as a "waveform" of the values of the 15 sensors (Santello and Soechting, 1997). The principal components (PCs) were then computed from the eigenvalues and eigenvectors of the matrix of the covariance coefficients between each of the 57 waveforms.

The principal components were ordered according to the amount of variance each component accounts for. The percentage of the total variance accounted for by each PC provided insight into the number of degrees of freedom. As we will show, the first 2 PCs accounted for $>80 \%$ of the variance. Because the higher-order PCs accounted for a very small percentage of the variance, the following question arose: are these higher-order PCs primarily noise (random variability), or do they actually contribute to differentiating among hand postures for different objects? To answer this question, we again resorted to the use of discriminant analysis and information theory. We first reconstructed hand postures for each trial using reduced sets of PCs. From these reconstructed postures, we generated confusion matrices and determined the amount of information that was provided by each of the PCs about the object that was grasped.

Hand postures were visualized using software (Persistence of Vision Ray Tracer) to render three-dimensional images of the hand. The angle at the distal interphalangeal (dip) joint was not measured in our experiments. For the sake of visualizing the entire hand posture, the amount of flexion at the dip joint was assumed to be $30 \%$ of the flexion at the pip joint of the same finger.

\section{RESULTS}

\section{Hand shaping}

Subjects adopted distinct hand shapes for imagined objects. Figure 1 shows the motion at each of the joints from one subject (U.H., five trials) during the transport phase of the movement to one particular object. In this case, a dictionary was to be grasped as if it were to be removed from a shelf. Movement time for each trial, which was typically $\sim 900 \mathrm{msec}$, has been normalized to 100 , i.e., the time between the "start" switch and the switch to signal the attainment of a static hand posture. From top to bottom, in the left column the traces depict the motion at the thumb (rot, flexion-extension at the mcp and ip joints, and abd) and abd between adjacent fingers (index-middle, middle-ring, and ringlittle, respectively). In the right column the traces depict the motion at the mcp and pip joints of each of the four fingers.

During the transport phase of the movement, abduction between the fingers typically increased (i.e., increasing values of the abd angles), as did the extension at the mcp joints of the four fingers (i.e., decreasing values of the mcp angles). Motion at the mcp joints then reversed direction before the hand attained a static posture. For this object, angular excursion at the pip joints tended to be monotonic. At the thumb, there was abduction and internal rotation. The range of motion at the thumb mcp and ip joints was generally much smaller.

The pattern illustrated in Figure 1 (which is generally representative of data obtained in this experiment) is qualitatively similar to that observed when subjects actually grasp objects (Paulignan and Jeannerod, 1996). In all cases, the hand aperture increases and then decreases before contact is made with the 


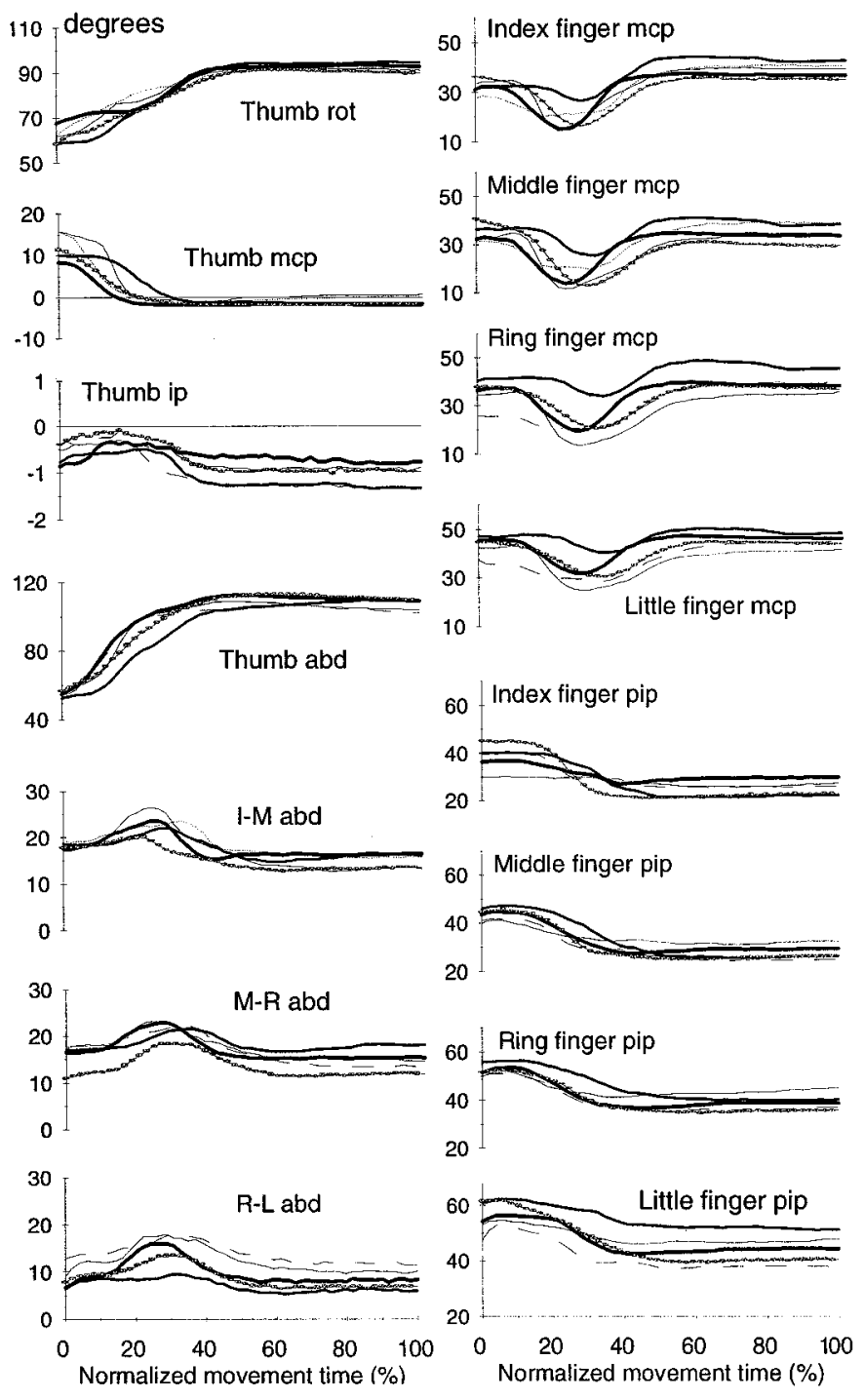

Figure 1. Time course of motion of the hand during a reaching and grasping movement to an imagined object. The traces depict data from five trials. In the left column, from top to bottom, the panels depict the motion of the thumb (rotation, flexion at the mcp and ip joints, and abduction) and the abduction angles between adjacent fingers: indexmiddle fingers $(I-M)$, middle-ring fingers $(M-R)$, and ring-little fingers $(R-L)$. In the right column, motion at the mcp and pip joints is depicted for each finger. Positive values denote flexion and abduction, respectively. At the thumb, positive values denote internal rotation. The data are for one subject (U.H.) who was instructed to grasp an imagined dictionary to remove it from a shelf. Time has been normalized from the onset of the movement, triggered by the release of a switch to the time at which the subject depressed a second switch to signal the attainment of a static posture. The static posture at the end of the movement was used in subsequent analysis.

object. Previous work has shown that hand shape gradually molds itself into the final posture (Santello and Soechting, 1998).

In the present study, we did not analyze the motion of the hand during the transport phase but instead confined our analysis to the static hand posture at the end of the trial. These were characterized by low intertrial variability in the joint angles (Fig. 1). For each joint angle, the mean SD (averaged across objects and subjects) ranged from 3 to $10^{\circ}$. Therefore, the hand postures in this experiment were fairly consistent, with an intertrial variability that was comparable in magnitude with that found just
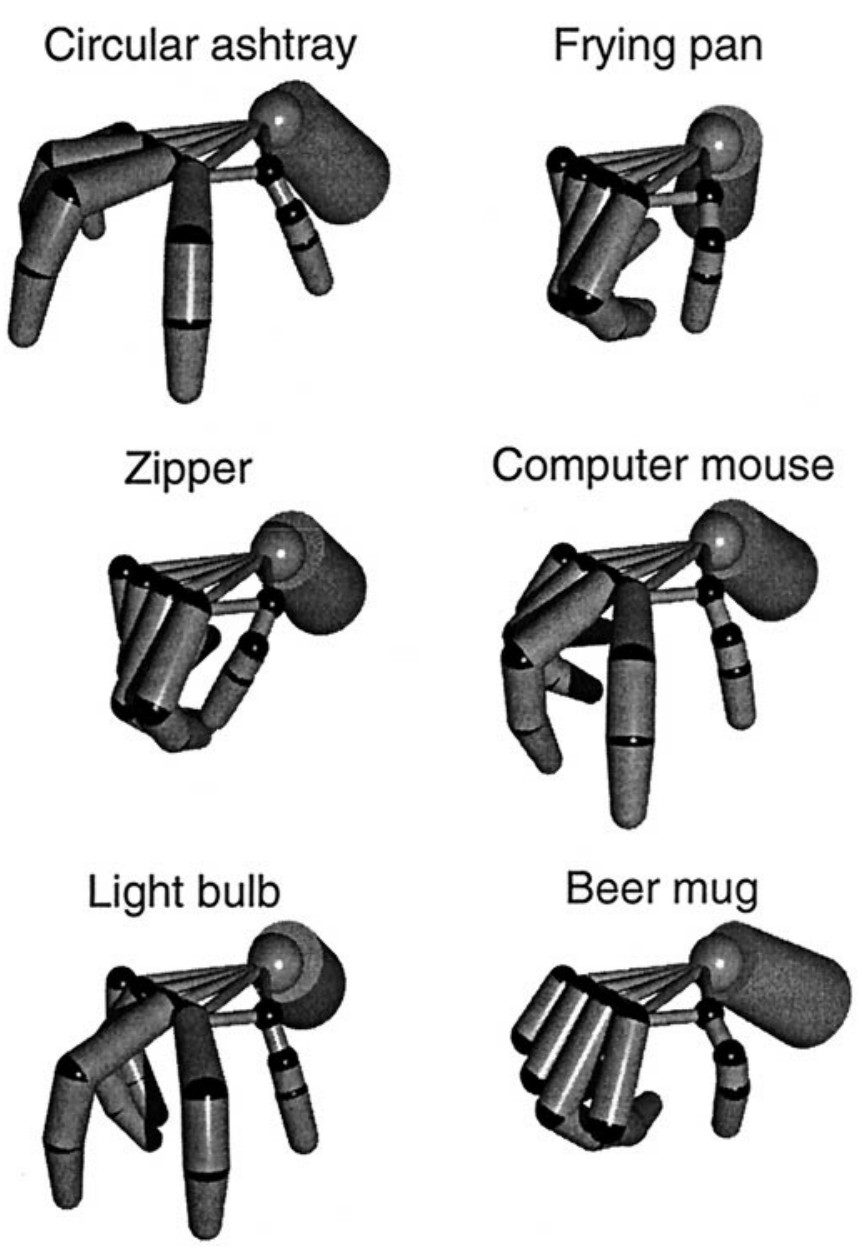

Figure 2. Hand postures for six different objects. The average hand postures produced by one subject for the six named objects have been rendered as three-dimensional images. Each of the three-dimensional images was rendered with the palm of the hand in the same orientation. Hence, the orientation as shown does not correspond to the actual orientation of the hand in space.

before contact when subjects grasped actual objects (Santello and Soechting, 1998), the latter ranging from 5 to $10^{\circ}$.

Figure 2 shows the average final hand postures from one subject (F.C.) for six different objects. To facilitate comparison between postures, these renderings are all shown from the same perspective, with the palm of the hand as the fixed reference. Therefore, the orientation of the hand postures illustrated does not reflect the actual orientation of the hand relative to the object. For example, subjects were instructed to grasp the "beer mug" by its handle, i.e., with the hand semipronated with respect to the object. The wrist would also be semipronated, but with additional ulnar deviation, when the "frying pan" is grasped by its handle, which was horizontal.

The hand postures in Figure 2 conform qualitatively to how one would expect the hand to be shaped if the object were physically present. Inspecting the renderings, one can guess the physical characteristics (shape and size) of the object in grasp, and they are consistent with named object. The postures are also clearly different from each other. To quantify this assertion, we computed discriminant functions to allocate data sets from individual trials to a particular object. These functions were used to generate confusion matrices from which the information transmitted by 


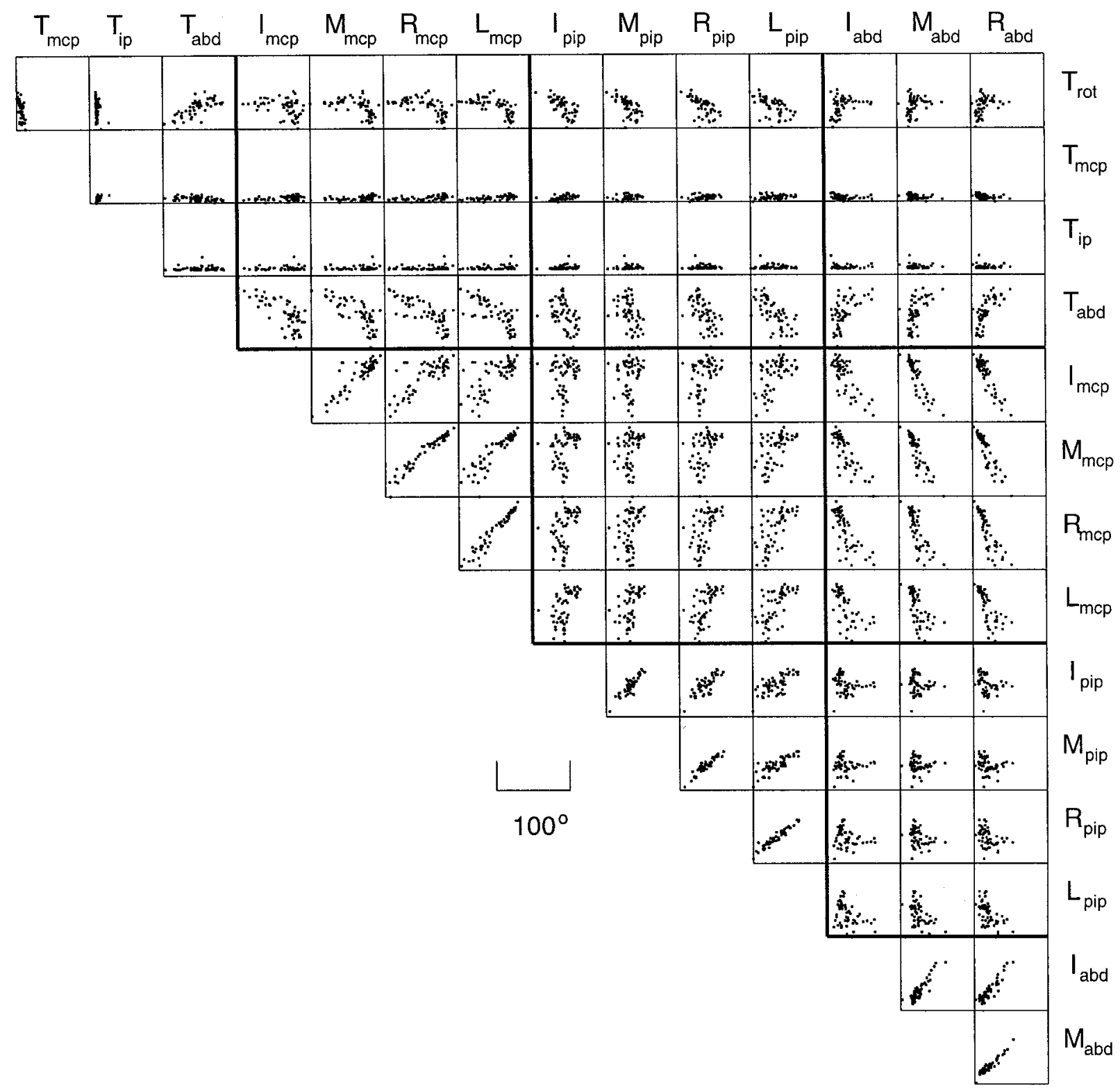

Figure 3. Patterns of covariation among the 15 joint angles of the hand. The average values of each of the joint angles for the 57 objects have been plotted against each other. The data are from one subject (M.F.). Note the strong covariation between mcp and pip angles at adjacent fingers (outermost diagonal), as well as the covariation between the abduction angles (bottom three elements) and the negative correlation between mcp and abd angles (last three columns).

hand posture about the object was computed. In particular we computed the SME index, expressed as a percentage of the maximum possible information that could be transmitted (i.e., if objects were predicted perfectly from hand shape). This index ranged from 77 to $88 \%$ across all subjects. For one subject (G.B.) we found that the range of angular excursion across hand postures was much lower than for the other subjects. For this subject, the value of the SME index was the lowest $(77 \%)$.

Thus, for most subjects hand posture transmitted $>80 \%$ of the maximum possible amount of information about objects, or 5 of the possible 5.8 bits of information $\left(5.8=\log _{2} 57\right)$. This corresponds to $2^{5}$ or 32 distinct postures. Therefore, more than half of the objects elicited a repeatable hand posture, whose features were distinct from those characterizing other postures.

\section{Patterns of covariation}

Not all of the joint angles of the hand were controlled independently of each other in this task. This can be observed by inspection of Figure 3, in which we have plotted the values of all of the joint angles against each other. The data are from one subject (M.F.) for all 57 objects. The extent to which posture varied differed considerably across the joint angles. Specifically, the range of motion at the mcp joints was $\sim 100^{\circ}$ and was almost as much for the pip and abd variables. However, motion at the thumb mcp and ip varied to a much lesser extent $\left(\sim 15\right.$ and $5^{\circ}$, respectively), the thumb being in an extended position for most of the objects. In contrast, thumb rot and abd were modulated over a wider range $\left(\sim 60\right.$ and $80^{\circ}$, respectively).

The angular excursion of the mcp joints of the four digits 

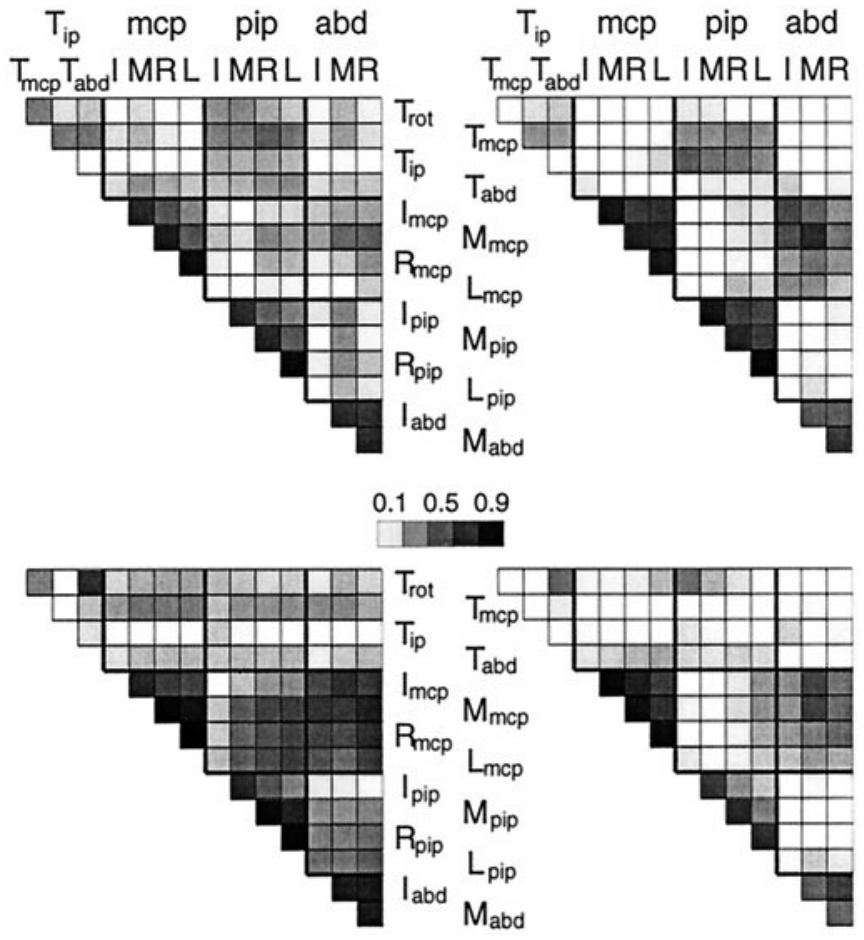

Figure 4. Coefficients of determination of the relations between joint angles of the hand. The gray scale in each square denotes the coefficient of determination $\left(r^{2}\right)$ for the relation between the angles indicated in the respective column and row. All but the data for the subject whose results are presented in Figure 3 are shown. Note the general similarity in the pattern for all subjects. The $r^{2}$ values shown were computed from pooled individual trials and are highly significant $(p<0.01$; $\mathrm{df}=283)$ for values $>0.02$.

tended to be positively correlated. Similarly, the pip angles tended to be positively correlated with each other. The extent of this correlation tended to be greatest for adjacent digits (Fig. 3, outermost diagonal). The abd angles of the fingers (Fig. 3, bottom three squares) were also positively correlated with each other. Finally, the abd angles tended to be negatively correlated with the mcp angles (Fig. 3, last three columns) and for this subject, there was a positive correlation between thumb abd and rot (Fig. 3, top row, third column). Excursions between other pairs of angles tended to covary to a much lesser extent. In particular, there was a large scatter in the data when the mcp and pip angles were plotted against each other.

The pattern illustrated in Figure 3 was generally similar to that of the other four subjects. This can be seen in Figure 4, which shows the coefficients of determination $\left(r^{2}\right)$ of the pairwise relationships among angles for these four subjects, plotted in the same format as Figure 3. In all subjects, mcp angles at adjacent fingers tended to be highly correlated, as were adjacent pip angles (Fig. 4, outer diagonal) and adjacent abd angles (Fig. 4, bottom three squares). The extent of correlation decreased as a function of the separation between pairs of fingers. There were also differences between the subjects. For example, the extent to which mcp and abd angles were negatively correlated with each other was greatest for the subject shown in Figure 3 and least for the subjects whose data are illustrated in the top left and bottom right panels of Figure 4 (last three columns).

\begin{tabular}{lllll}
\hline \multicolumn{5}{l}{ Table 2. Percent variance accounted for by each principal component } \\
Subjects & $\mathrm{PC}_{1}$ & $\mathrm{PC}_{2}$ & $\mathrm{PC}_{3}$ & $\mathrm{PC}_{4}$ \\
\hline $\mathrm{FC}$ & 52.9 & 24.7 & 8.4 & 4.8 \\
$\mathrm{~GB}$ & 49.5 & 37.6 & 4.8 & 4.6 \\
$\mathrm{MF}$ & 74.8 & 13.0 & 5.4 & 2.9 \\
$\mathrm{MS}$ & 79.3 & 10.0 & 5.0 & 2.2 \\
$\mathrm{UH}$ & 62.9 & 17.2 & 8.6 & 5.9 \\
\hline
\end{tabular}

\section{Defining postural synergies}

The results of the analysis presented in Figures 3 and 4 indicated that not all of the joint angles of the hand are controlled independently of each other in shaping the hand to grasp different objects. This implies a reduction in the number of degrees of freedom, and PC analysis was used to identify the effective degrees of freedom more precisely.

On average, the first three PCs accounted for $\sim 90 \%$ of the variance, with the first two PCs accounting for $\sim 84 \%$ (Table 2), suggesting a substantial reduction in the number of degrees of freedom, from 15 to 2 or 3 degrees of freedom. Furthermore, as shown in Figure 5, there was a high degree of consistency in the first two PCs across subjects (but see below for subject G.B.). Figure 5 illustrates the amount by which each of the angles changes for a unit change in the amplitude of the first (left column) or the second (right column) PC. The top panels show results for all of the subjects. Positive values denote flexion and abduction. Internal rotation of the thumb is also denoted by positive values.

Initially, there was a high degree of similarity in the waveforms of the first two PCs for all subjects except subject GB. However, we found that rotating the PC axes (Flanders and Herrmann, 1992) for this subject greatly improved the correlation with the PCs from the other subjects, as can be seen by comparing the open symbols in Figure 5 (top panel) with the data for the other four subjects. The similarity among the first two PCs was quantified by regression analysis. There were strong correlations for the first and second PCs, with $r^{2}$ values ranging from 0.79 to 0.97 for the first PC and from 0.34 to 0.90 for the second PC. The intersubject correlations for higher PCs, however, were weak.

The bottom panels of Figure 5 show the amplitude of the first two PCs averaged across four subjects (excluding G.B.). The shading indicates values above zero (flexion and abduction) and below zero (extension and adduction). The first PC was characterized by flexion at all the mcp joints and a lesser degree of flexion at all the pip joints (dark shading), by adduction at all fingers, and by thumb external rotation and adduction (light shading). This kinematic profile can also be related to the results illustrated in Figures 3 and 4, showing high degrees of pairwise correlations between all the mcp joints and similarly for the pip and abd angles. With regard to the thumb, the excursion of the thumb mcp and ip angles provided by the first PC (Fig. 5, left column) is less than that for thumb rotation and abduction. This is also consistent with Figure 3, in which the range of motion among the four thumb angles is clearly different.

In contrast, the second PC was characterized by extension at the mcp joints (Fig. 5, light shading) and flexion at the pip joints (Fig. 5, dark shading), with little modulation of finger abduction. In the second PC, the thumb angles had the same pattern of modulation as they did for the first PC.

The postural synergies implied by the first two principal com- 

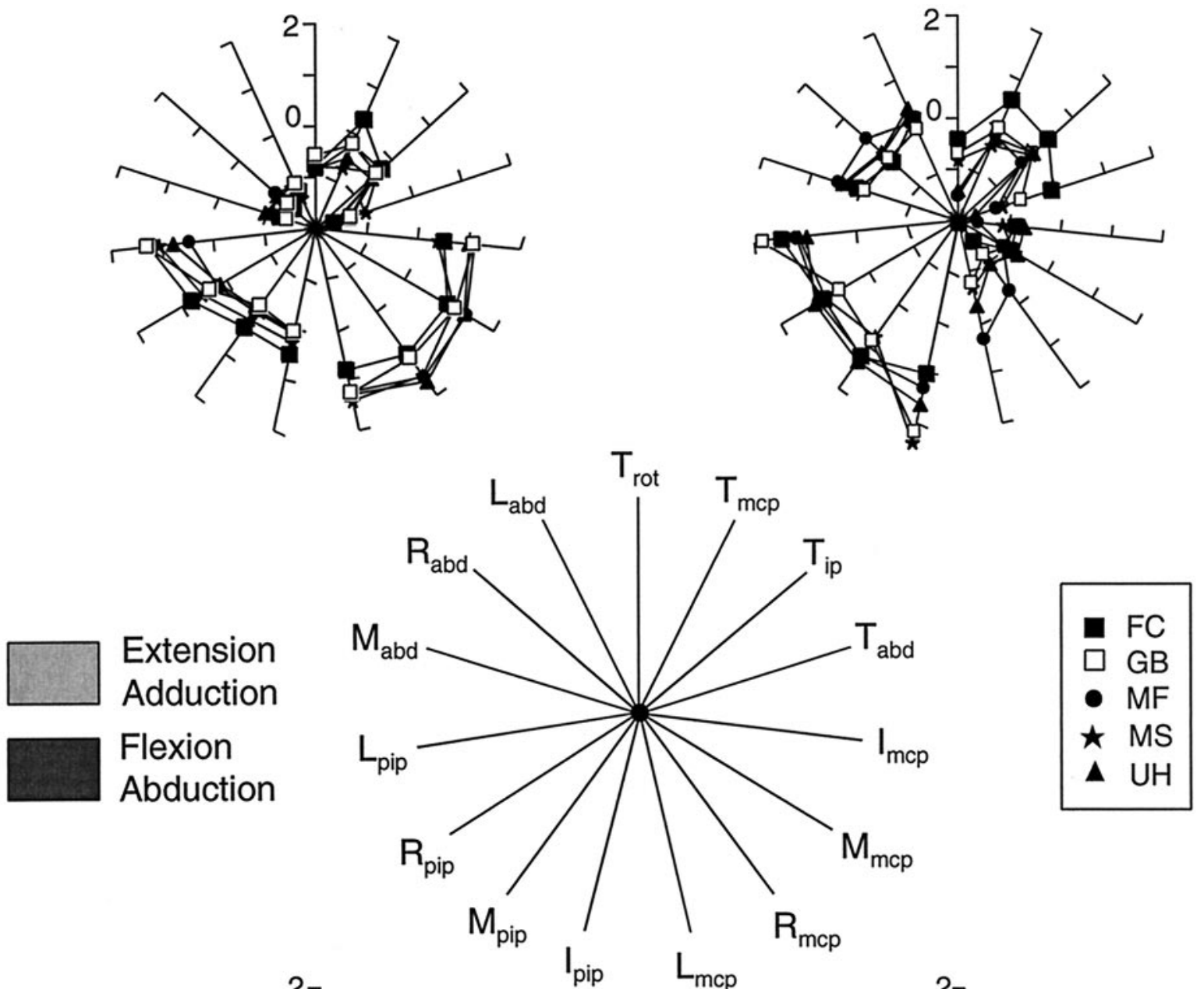

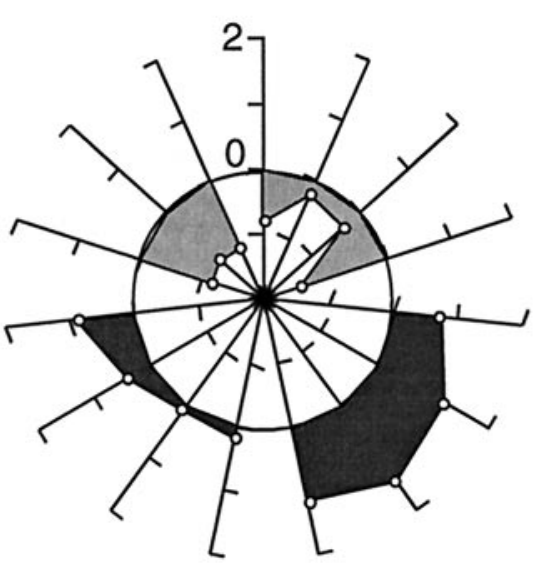

\section{1st Principal Component}

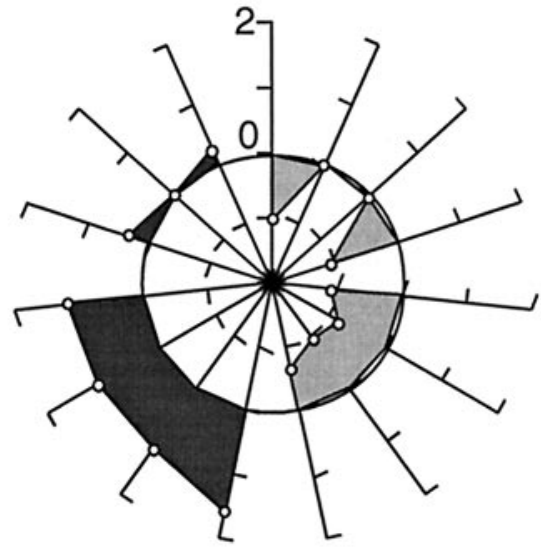

\section{2nd Principal Component}

Figure 5. Waveforms of the first two principal components. Top panels, Change in each of the joint angles (in degrees) resulting from a unit change in the first and second PCs (left and right sides, respectively) for all five subjects. The values are shown in their normalized form. The data for one subject (G.B., open symbols) were obtained by first rotating the $\mathrm{PCs}\left(\mathrm{PC}_{1}^{*}=\mathrm{PC}_{1} \cos \theta+\mathrm{PC}_{2} \sin \theta ; \mathrm{PC}_{2}^{*}=-\mathrm{PC}_{1} \sin \theta+\mathrm{PC}_{2} \cos \theta ; \theta=128^{\circ}\right)$. Bottom panels, Amplitudes of each PC averaged across all subjects except subject G.B. The shading indicates values above and below zero. Positive values denote flexion and abduction. At the thumb, positive values denote internal rotation.

ponents are depicted in Figure 6. This figure diagrams the threedimensional hand postures along the $\mathrm{PC}_{1}$ and $\mathrm{PC}_{2}$ axes reconstructed from the data for one subject (U.H.). The hand posture in the center of the PC axes was rendered using the average of 57 hand postures. The other four postures were computed by adding the minimum or maximum values of $\mathrm{PC}_{1}$ and $\mathrm{PC}_{2}$ to the average hand posture (for which the values of the $\mathrm{PC}$ coefficients are all zero). 


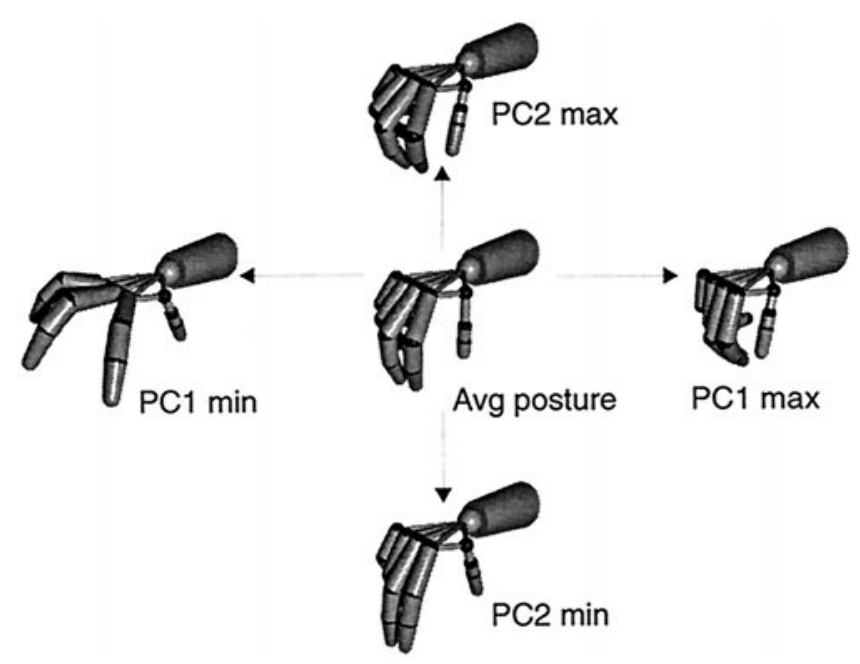

Figure 6. Postural synergies defined by the first two principal components. The hand posture at the center of the PC axes is the average of 57 hand postures for one subject (U.H.). The postures to the right and left are for the postures for the maximum ( $\max$ ) and minimum ( $\min$ ) values of the first principal component $(P C 1)$, coefficients for the other principal components having been set to zero. The postures at the top and bottom are for the maximum and minimum values of the second principal component $(P C 2)$.

Along the $\mathrm{PC}_{1}$ (horizontal) axis, at one extreme the fingers are extended at the mcp joint and abducted $\left(\mathrm{PC}_{1} \mathrm{~min}\right)$. At the other extreme, they are flexed at the mcp joint and adducted $\left(\mathrm{PC}_{1} \max \right)$. The excursion at the pip joints remains approximately constant. At the thumb, moving toward $\mathrm{PC}_{1}$ max, abduction and internal rotation decrease. These angular changes can be visualized in Figure 6 as a gradual closure of the hand. Along the $\mathrm{PC}_{2}$ (vertical) axis, the changes in angular excursion are of a smaller amplitude: moving toward $\mathrm{PC}_{2}$ max, the pip joints flex, whereas the mcp joints extend. As was the case for $\mathrm{PC}_{1} \max$, the thumb abduction and internal rotation decrease.

Figure 7 shows how the hand postures for the 57 objects (for one subject, M.F.) were distributed in the plane of the first two PCs. It is clear that the hand postures did not tend to cluster into a few discrete groups. This feature was common to all subjects. The fact that no distinct groups of postures were found indicates that the modulation of hand posture occurred in a gradual manner along a continuum in a multidimensional (at least twodimensional) space. Furthermore, objects that would be grasped with precision and power grips were not segregated. For example, for this subject, "chalk" (grasped with a precision grip) and "wrench" (grasped with a power grip) are nearest neighbors in PC space.

In Figure 7, the coefficients of the first two PCs seem to be aligned on two main axes. These axes are approximately orthogonal to each other: one with a negative slope, the other with a positive slope and intersecting near the origin of the $\mathrm{PC}_{1}$ axis. Piecewise linear regression analysis confirmed this impression; breaking the data into two groups significantly improved the fit. This feature was also seen in other two subjects (G.B. and M.S.). For all three subjects, there was considerable scatter in the data about these lines, with low $r^{2}$ values, ranging from 0.21 to 0.35 . The slopes of the two lines, however, were significantly different from zero $(p<0.05)$ for all three subjects. For the other two subjects, the data were more uniformly distributed in the plane of the first two PCs.
The bilinear fit to the data of Figure 7 is shown in Figure 8. To illustrate how the hand postures varied along the axes defined by the regression lines, the actual hand postures for five objects are shown at the extremes of the fit, at its midpoints, and at the break point. One may consider the two lines in Figure 8 to represent two separate synergies for hand posture for this subject. The left line has a negative slope, and the change in the weighting of the two PC coefficients will have an opposite sign as one progresses along the line. By referring to Figure 5, one can see that the changes at the mcp and abd angles attributable to $\mathrm{PC}_{1}$ and $\mathrm{PC}_{2}$ will reinforce each other, but that those at the pip joints are partially canceled, as are those at the thumb's joint angles. Thus this first synergy would correspond primarily to a combination of extension at the mcp finger joints coupled with abduction of all fingers. This is clear by inspecting the renderings of the postures for the three objects ("compact disk" to "light bulb" to "espresso cup"). Along the second line, the slope is positive, and, accordingly, so is the weighting of the first two PC coefficients. By inspection of Figure 5, this suggests a cancellation of their respective effects for the mcp and abd angles, with most of the motion (flexion) occurring at the pip joints of the fingers, concurrent with internal rotation and adduction of the thumb. Thus, the aperture of the hand decreases in the progression from "espresso cup" to "wrench" to "bucket."

A qualitatively similar pattern was also found for two other subjects (M.S. and G.B.). At one extreme of the fit, the hand was at its maximum aperture, the object grasped being a "compact disk." For these subjects, the break point of the fit occurred at "ice cube" or "cherry" (M.S.) and "pen" (G.B.), whereas "fishing rod" and "rope" were located at the other extreme. The finding that a gradual modulation of hand posture could be detected along two main axes in PC space points to the possible existence of two main synergies through which hand shape is modulated according to different objects' features.

\section{How many effective degrees of freedom are there?}

The results of the principal components analysis presented so far indicate that the first two PCs accounted for $>80 \%$ of the variance in hand posture and in three of the five subjects for $>87 \%$ of the variance. This result can be taken to suggest that the control of hand posture involves principally two synergies, manifested either singly or in combination. These could correspond to the axes of the first two PCs (Fig. 6) or to axes oriented obliquely in the plane of these PCs (Figs. 7, 8).

This interpretation appears to be somewhat at variance with the data shown in Figures 3 and 4, where there was a high degree of correlation only among a subset of the joint angles (principally of the mcp and pip joints of adjacent joints and the abd angles of the fingers). There were also many instances in which pairs of joint angles were only poorly correlated, suggesting that there are more than two effective degrees of freedom for the control of hand posture and that several higher-order PCs would also be needed to represent this rather limited covariation in joint angles.

There are two alternative solutions to this paradoxical result: (1) higher-order PCs are needed but represent noise in the system; and (2) the higher-order PCs do in fact contribute to discriminating among hand shapes for different objects. The latter possibility would suggest that the higher-order PCs represent additional effective degrees of freedom that are controlled by the nervous system. To study this issue in more detail, we performed additional analysis on the PCs, using discriminant analysis and information theory. First, we reconstructed the hand 


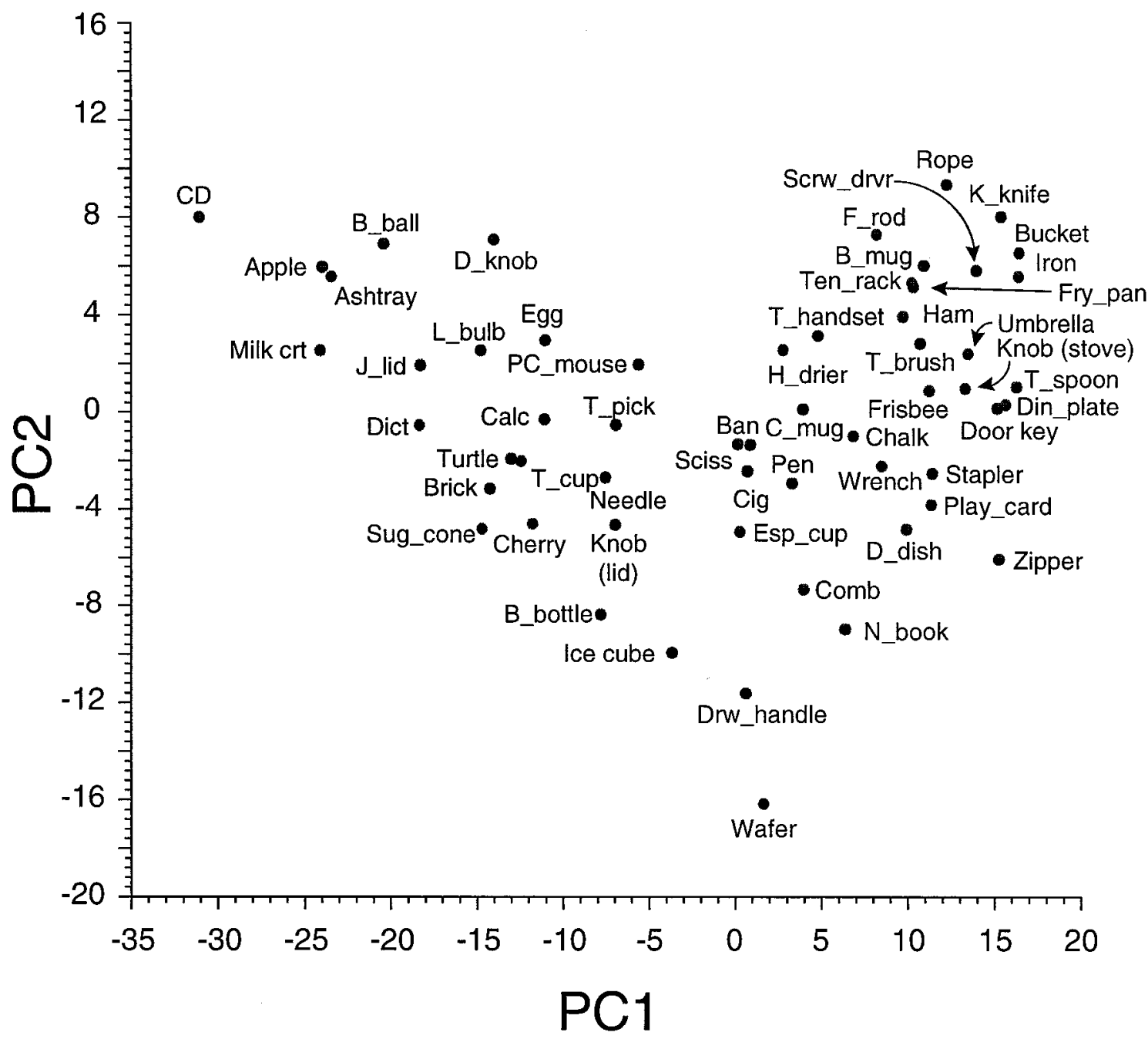

Figure 7. Distribution of hand postures in the plane of the first two principal components. The coefficients of the first two principal components are shown for each of the 57 objects for one subject (M.F.). Note the lack of clustering and the distribution of the coefficients along two main axes.

postures using an increasing number of PCs, i.e., the first, the first plus the second, and so forth up to 14 PCs. (The amplitude of the 15 th PC was found to be approximately zero.) We then determined how much information about the objects increased as the number of degrees of freedom (PCs) increased. If the higherorder PCs represent noise (random variability), the information transmitted by hand shape about the object should not increase (and may actually decrease) when higher-order PCs are used to define hand posture. Conversely, if the higher-order PCs do contribute to discriminating among hand shapes, the information transmitted should increase as more PCs are included.

The results of this analysis are illustrated in Figure 9. The amount of information continued to increase monotonically up to at least the fifth or the sixth PC, even though these higher-order principal components contributed little to the variance (Table 2). Clearly, more than two degrees of freedom are used to mold the hand into the shape appropriate to grasp a particular object, and the higher-order PCs do not simply represent random variability.

Given that higher-order PCs do not simply represent noise, it is possible that the hand postures associated with a few of the objects might be best represented by higher PCs, i.e., that the amplitude of the higher-order coefficients might be substantial for one or a few objects. Thus, the overall variance attributed to one
PC might be small, but its contribution to a few postures might be large. If this were the case, the distribution of the PC coefficients for the 57 objects would be multimodal and/or have a broad range. Figure 10 shows that this is not the case. Shown is the distribution of the amplitudes of the first five PC coefficients for one subject (U.H.). (The amount of variance accounted for by each PC is noted below each histogram.) The amplitude of each of the coefficients has been normalized relative to the maximum (or minimum values) of the first PC. The coefficients for the first and second PCs were widely distributed, the range of values for the first $\mathrm{PC}$ being greater than the range of the second. Although the distributions of the amplitudes of the $\mathrm{PC}_{3}, \mathrm{PC}_{4}$, and $\mathrm{PC}_{5}$ coefficients were not statistically normal, they were not multimodal, and the range of amplitudes was small. Hence, higher PCs do not seem to contribute substantially to any one particular hand posture. These features were also found in the other subjects. This finding implies that the amplitudes of higher-order coefficients were generally small, irrespective of the object.

Despite the very small $\mathrm{PC}_{3}-\mathrm{PC}_{5}$ coefficients for all postures, it is nevertheless possible that for some objects, there is a substantial difference between the joint angles computed from the first two PCs and the actual posture at one or a few joints. (Small $\mathrm{PC}_{3}-\mathrm{PC}_{14}$ coefficients could potentially summate for one angle 


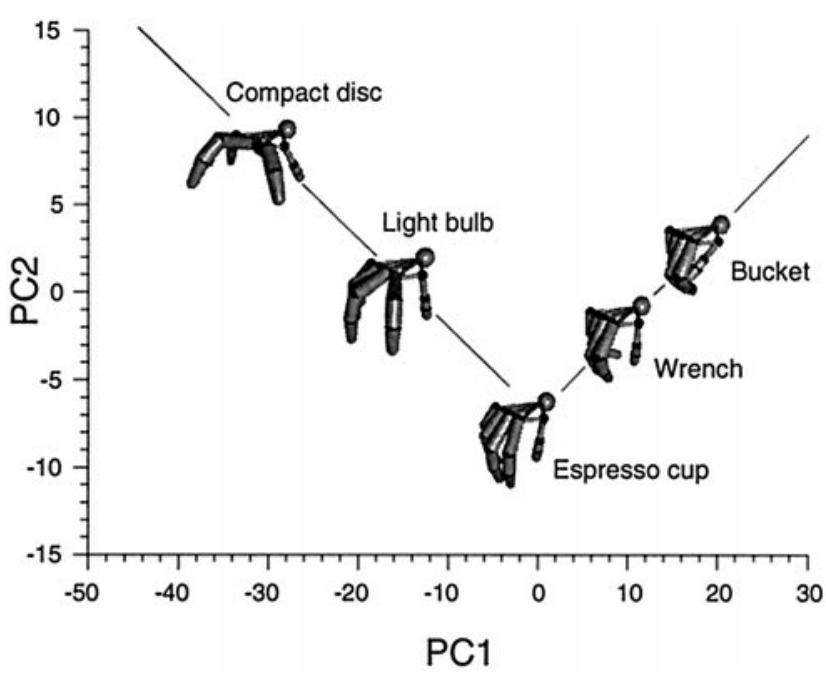

Figure 8. Grasping synergies. The two lines show the results of a bilinear fit to the data in Figure 7. Superimposed on these lines are hand postures for five of the objects shown at locations that correspond to the values of the first two PC components. Note the flexion at the mcp joint and adduction of the fingers as one descends the line at the left and the closure of finger aperture achieved by flexion at the pip joints of the fingers and thumb adduction and internal rotation as one ascends the line at the right.

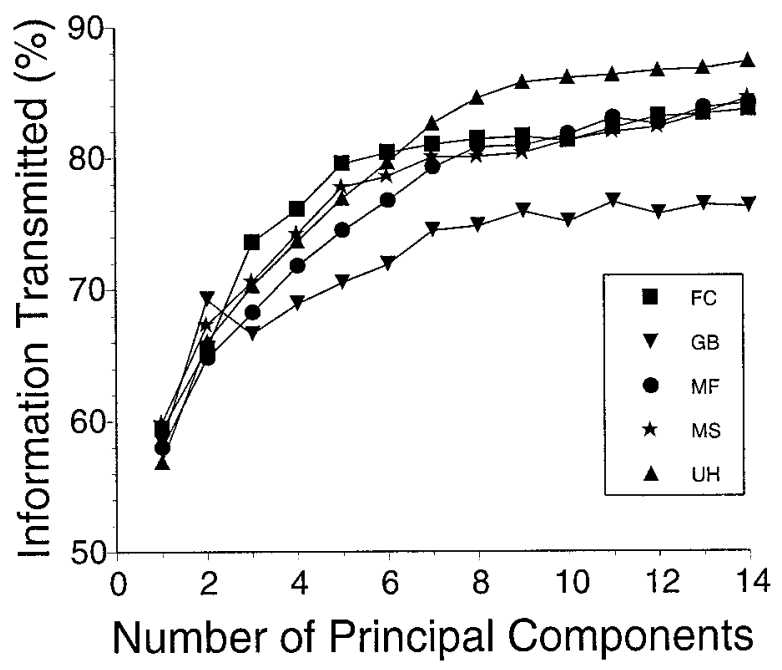

Figure 9. Information transmitted by each of the PCs about the "object" in grasp. The SME (the percent of the information possible) is plotted against the number of PCs used to reconstruct hand postures for each of the subjects. The amount of information increases until the fifth to the sixth PC is added.

and cancel each other at the other angles.) If that were the case, one would expect a multimodal distribution in the errors at the joint angles predicted from the first two PCs compared with the actual posture.

To address this question, we initially focused our attention on the first three PCs, reconstructing the hand postures from the two or three PCs and comparing the reconstruction to the measured hand posture. The results of this analysis are shown in Figure $11 \mathrm{~A}$ for one object ("cherry") and one subject (M.F.). The object was chosen because the hand postures reconstructed using only two PCs were readily confused with those for other objects on two of five trials, whereas this object was discriminated perfectly (on five of five trials) when three or more PCs were used. The bar graph shows the errors in predicting each of the angles when either two or three PCs are used to reconstruct the posture of the hand. In this instance, the third PC diminished the error at three of the mcp joints and for thumb abd by $\sim 5-15^{\circ}$. Beyond the third PC, the errors were small and uniformly distributed.

Figure $11 B$ shows the distribution in the errors when hand posture was reconstructed from the first two PCs for one subject (M.F.) and for all objects and all joint angles. It is clear that for the majority of cases $(\sim 78 \%)$, the mismatch lies in the range of $\pm 5^{\circ}$ and only rarely exceeds $10^{\circ}$. Thus, the example illustrated in Figure $11 A$ is one that occurred rarely. Similar observations were made for the other subjects as well. Thus, the increase in the information transmitted by higher-order PCs does not come about because they effect large changes in select joint angles for select objects. Instead, their contribution appears to be more subtle.

\section{DISCUSSION}

The kinematic analysis of hand postures for grasping objects showed that there was a considerable reduction in the number of degrees of freedom. In particular, principal components analysis showed that two principal components were able to account for $>80 \%$ of the variance in the data and that the variance contributed by other principal components was small. This result can be interpreted to imply that there are two fundamental synergies governing the manner in which the hand is shaped to grasp objects. However, an analysis based on information theory led to a somewhat different interpretation, because information about the object that was provided by hand shape gradually increased when higher-order principal components (up to the fifth or sixth and beyond) were included (Fig. 9), suggesting that there are at least five to six effective degrees of freedom. Finally, we found that hand shapes did not cluster, nor was there any particular correlation between the final shape of the hand and the type of grip (e.g., precision or power) that would be used in using the object. In the following, we will take up these findings in some detail, but first we will comment briefly about the design of the motor task we used.

\section{Methodological considerations}

We asked subjects to imagine that they were grasping a set of common objects to put them to their intended use. We did not use actual objects, because the posture of the hand, when grasping an actual object, can be a consequence of central control signals as well as of the mechanical interaction of the hand with the object. We needed to have a task in which posture would not be confounded by the latter. We preferred not to measure posture just before contact with the object, as in a task in which the subject is asked to reach to and lift a set of objects (Johansson and Cole, 1992; Santello and Soechting, 1998). The manner in which an object (such as a "teaspoon" or a pair of "scissors") is grasped to lift it from a horizontal surface may be quite different from the manner in which it is held when it is put to its intended use.

We do not know to what extent subjects reproduced postures they would have assumed had they grasped the actual object, partly because we asked them to rely on their memory of familiar objects. However, the hand shapes that they assumed generally conformed to the shape one would expect (Figs. 2, 8), and the intertrial variability in joint angles was small. We believe an important aspect of the experimental design was that we encouraged subjects to incorporate motion of the proximal arm into the shaping of the hand. Even though the control of proximal and 


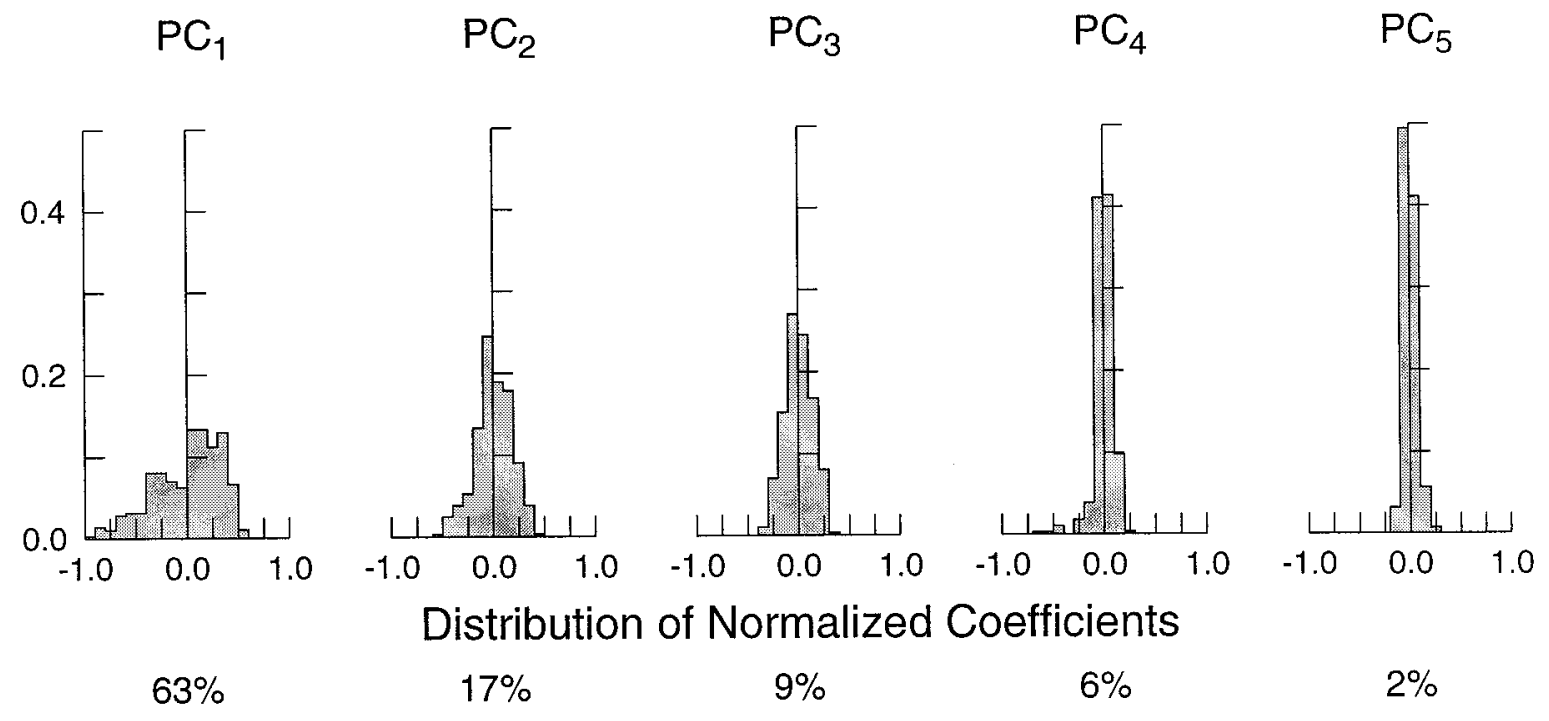

Figure 10. Distribution of normalized amplitudes of the first five principal components. The amplitudes of the first five PCs have been normalized to the maximum (or minimum) value of the first PC. The data shown are for one subject (U.H.). Note that the amplitudes of the third through the fifth PCs are uniformly small, even though they contribute substantially to the information transmitted (Fig. 9).
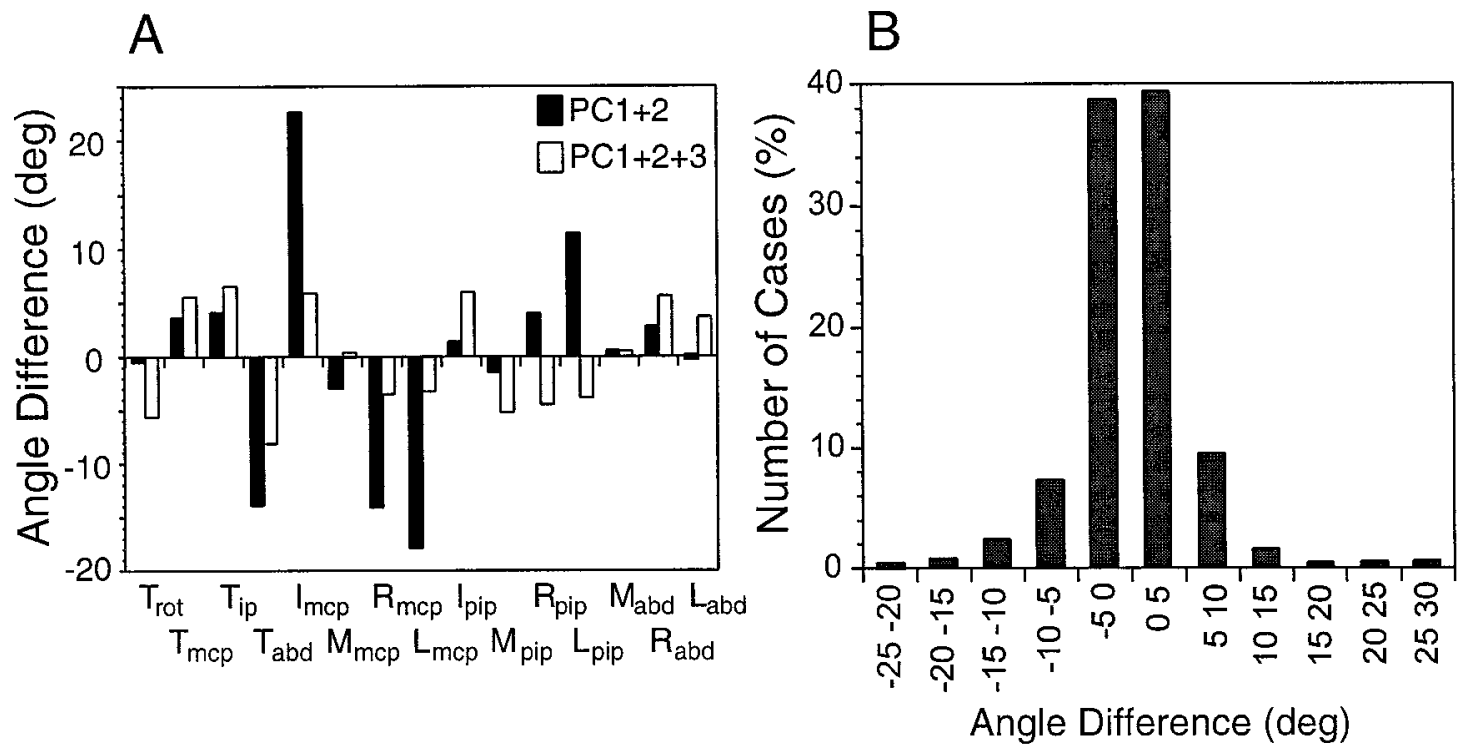

Figure 11. Difference between actual hand posture and postures reconstructed from PCs. $A$, Angular difference at each of the joint angles between the actual posture of the hand and the posture reconstructed from the first two or three PCs for one object (cherry) and one subject (M.F.). $B$, Distribution of the angular differences for all joint angles between hand postures reconstructed from the first two PCs and the actual postures recorded. The data are for all objects from one subject (M.F.). $T$, Thumb; $I$, index finger; $M$, middle finger; $R$, ring finger; $L$, little finger.

distal parts of the arm may evolve in parallel when subjects grasp an object (Paulignan and Jeannerod, 1996), the two are not independent of each other (cf. Soechting and Flanders, 1993).

\section{Synergies for the control of the hand}

As already mentioned above, two principal components could account for $>80 \%$ of the variance. Furthermore, the waveforms of these two principal components were highly consistent for four of the five subjects. (The fifth subject's results also conformed after rotation of the PC axes.) Finally, detailed analysis of how the individual joint angles of the hand are related to these two principal components (Figs. 5, 6, 8) yielded results that are readily interpreted as postural synergies-for example, one that combines flexion at the mcp joints with adduction at all fingers, and a second combining flexion at the pip joints with internal rotation and adduction of the thumb to control finger span (Fig. 6). Our results suggest that these synergies can manifest themselves individually (as in Fig. 6) or in combination (Fig. 8). Such a combination is reminiscent of the concept of "flexible synergies" proposed by Macpherson (1991).

This picture is incomplete, however. Each of the higher-order principal components (e.g., numbers 3-6) contributed only a small amount to the overall variance. Even when the data were analyzed object by object, the amplitude of these components was generally small (Fig. 10), as was the change in angular excursion that they contributed at each joint (Fig. $11 B$ ). Nevertheless, these higher-order principal components did not represent mostly 
noise. In fact they contributed substantially to the information that hand shape provided about the object that was "grasped" (Fig. 9).

This observation suggests the following interpretation. The control of hand shape is effected at two levels. Superimposed on a coarse control of hand shape, which manifests itself in a few distinct patterns of coordination of all of the joints of the hand, is a mode of control that may affect the joints at a finer level. Because the higher-order principal components were very small and were not consistent from subject to subject, we were not able to characterize this "finer level of control" more precisely. The higher-order PCs had coefficients that were distributed among all of the joint angles, suggesting that this finer control is also distributed. However, the principal components per se need not have any physical significance. Conceivably, a linear combination of several PCs could yield a pattern of motion restricted to one finger or perhaps even one joint.

This hypothesis is consistent with the observation with which we began this paper, namely that a disproportionate amount of sensorimotor cortical area is devoted to the hand. It is also consistent with previous demonstrations (Schieber, 1991; Soechting and Flanders, 1997) of a tendency for coordinated motion of the fingers. These previous studies also found that this was merely a statistical tendency and that it was not obligatory. We do not know whether these two hypothesized levels of control are subdivided by anatomical distinctions. However, a certain extent of covariation in the amplitude of finger movement is attributable to the biomechanical arrangement of the extrinsic finger muscles and the patterning of co-activation of these muscles (Maier and Hepp-Reymond, 1995; Schieber, 1995). The finer level of control may be required to override this musculoskeletal and neuromuscular coupling.

\section{The relationship between hand shape and contact force}

As already noted, we could find no evidence for a clustering of the static postures for the various objects (Fig. 7), even though we were careful to select objects that would normally be grasped with a wide variety of grips. Furthermore, objects that elicited similar hand shapes were often associated with grips that were quite distinct (i.e., precision vs power grips), and objects that are considered to be held in a power grip could elicit hand shapes that were objectively quite dissimilar (Fig. 7). This observation does not imply a refutation of the previous attempts at classifying hand grips described in the introductory remarks. As was mentioned there, all of these schemes are based on considerations of which finger(s) and which part(s) of the finger(s) contact and exert force on the object. That is to say, the schemes are based on the control of contact force, rather than posture.

Our observations suggest that the control of static hand posture (i.e., kinematics) is separate from the control and regulation of contact force. Clearly, the two are not independent, because the hand must be shaped properly so that the correct set of fingers makes contact with the object. However, our results imply that there is no one-to-one relation between posture and force control. For example, very different contact forces may be exerted with the hand in the same posture, depending on the object that is in grasp (Fig. 7). This suggestion is consistent with recent observations of neural activity in the hand area of primary motor cortex, which suggest a dissociation between the neural correlates of force and of kinematics in a task requiring monkeys to control the grasp force of variously shaped objects (Gomez et al., 1997). The suggestion is also consistent with the very different sensory demands of the control of contact force and the control of posturethe former is exquisitely dependent on tactile feedback (Johansson and Cole, 1992; Johansson et al., 1992a,b).

\section{REFERENCES}

Cutkosky MR, Howe RD (1990) Human grasp choice and robotic grasp analysis. In: Dextrous robot hands (Venkataraman ST, Iberall T, eds), pp 5-31. New York: Springer.

Elliott JM, Connolly KJ (1984) A classification of manipulative hand movements. Dev Med Child Neurol 26:283-296.

Flanders M, Herrmann U (1992) Two components of muscle activation: scaling with the speed of arm movement. J Neurophysiol 67:913-943.

Glaser EM, Ruchkin DS (1976) Principles of neurobiological signal analysis. New York: Academic.

Gomez JE, Mason CR, Ebner TJ (1997) Responses of precentral cortical neurons during whole hand grasping. Soc Neurosci Abstr 23:1555.

Iberall T, MacKenzie CL (1990) Opposition space and human prehension. In: Dextrous robot hands (Venkataraman ST, Iberall T, eds), pp 32-54. New York: Springer.

Iberall T, Bingham G, Arbib MA (1986) Opposition space as a structuring concept for the analysis of skilled hand movements. Exp Brain Res Ser 15:158-173.

Johansson RS, Cole KJ (1992) Sensory-motor coordination during grasping and manipulative actions. Curr Opinion Neurobiol 2:815-823.

Johansson R, Häger C, Bäckström L (1992a) Somatosensory control of precision grip during unpredictable pulling loads. III. Impairments during digital anesthesia. Exp Brain Res 89:204-213.

Johansson R, Häger C, Riso R (1992b) Somatosensory control of precision grip during unpredictable pulling loads. II. Changes in load force rate. Exp Brain Res 89:192-203.

Johnson KO, Phillips JR (1981) Tactile spatial resolution. I. Two-point discrimination, gap detection, grating recognition. J Neurophysiol 46:1177-1191.

Johnson RA, Wichern DW (1992) Applied multivariate statistical analysis. Englewood Cliffs, NJ: Prentice Hall.

Kamakura N, Matsuo M, Ishii H, Mitsuboshi F, Miura Y (1980) Patterns of static prehension in normal hands. Am J Occup Ther 7:437-445.

Klatzky RL, Pellegrino J, McCloskey B, Doherty S, Smith T (1987) Knowledge about hand shaping and knowledge about objects. J Mot Behav 19:187-213.

Macpherson JM (1991) How flexible are muscle synergies?. In: Motor control: concepts and issues (Humphrey DR, Freund H-J, eds), pp 33-47. Chichester, UK: Wiley.

Maier MA, Hepp-Reymond M-C (1995) EMG activation patterns during force production in precision grip. II. Muscular synergies in the spatial and temporal domain. Exp Brain Res 103:123-136.

Napier JR (1956) The prehensile movements of the human hand. J Bone Joint Surg 38B:902-913.

Paulignan Y, Jeannerod M (1996) Prehension movements. The visuomotor channels hypothesis revisited. In: Hand and brain. The neurophysiology and psychology of hand movements (Wing AM, Haggard P, Flanagan JR, eds), pp 265-282. San Diego: Academic.

Sakitt B (1980) Visual-motor efficiency (VME) and the information transmitted in visual-motor tasks. Bull Psychol Soc 16:329-332.

Santello M, Soechting JF (1997) Matching object size by controlling finger span and hand shape. Somatosens Motor Res 14:203-212.

Santello M, Soechting JF (1998) Gradual molding of the hand to object contours. J Neurophysiol 79:1307-1320.

Schieber MH (1991) Individuated finger movements of Rhesus monkeys: a means of quantifying the independence of the digits. J Neurophysiol 65:1381-1391.

Schieber MH (1995) Muscular production of individuated finger movements: the roles of extrinsic finger muscles. J Neurosci 15:284-297.

Shannon CE (1948) The mathematical theory of communication. Bell Syst Techn J 27:379-423.

Soechting JF, Flanders M (1993) Parallel, interdependent channels for location and orientation in sensorimotor transformations for reaching and grasping. J Neurophysiol 70:1137-1150.

Soechting JF, Flanders M (1997) Flexibility and repeatability of finger movements during typing: analysis of multiple degrees of freedom. J Comput Neurosci 4:29-46. 